\title{
EXISTENCIA Y UNICIDAD DE LA SOLUCIÓN GENERALIZADA DE UNA ECUACIÓN DIFERENCIAL HIPERBÓLICA QUE MODELA LA PROPAGACIÓN DE UNA ONDA EN UN MEDIO ELÁSTICO SOMETIDA A UNA FUERZA EXTERNA
}

\author{
EXISTENCE AND UNITY OF THE GENERALIZED SOLUTION OF A HYPERBOLIC \\ DIFFERENTIAL EQUATION THAT MODELS THE PROPAGATION OF A WAVE IN A \\ ELASTIC MEDIUM SUBJECT TO AN EXTERNAL STRENGTH
}

\author{
Jhony Alfonso Chávez Delgado', Luis César Méndez Avalos², \\ Eduardo Rodríguez Delgado, Luis Asunción López Puycán ${ }^{4}$
}

\section{RESUMEN}

El propósito de este artículo fue investigar, cómo con el desarrollo del análisis funcional y la teoría de distribuciones se pueden establecer soluciones generalizadas que permiten resolver ecuaciones hiperbólicas que modelan la propagación de una onda en un medio elástico sometida a una fuerza externa. Desde un punto vista físico, este modelo hiperbólico requiere que la existencia de una solución sea físicamente aceptable y "controlable". Matemáticamente esto se traduce en problemas de existencia, unicidad y dependencia continua de las condiciones iniciales y de contorno. Se empleó para el desarrollo del artículo el método lógico deductivo para demostrar la existencia y unicidad de la solución generalizada del problema de evolución hiperbólica, que consistió en aproximar la solución del problema por autofunciones lineales de dimensión finita para luego construir un subespacio denso en un espacio de Hilbert separable. Luego lo dividimos en etapas: Acotación de las soluciones aproximadas, convergencia de las soluciones aproximadas y verificación de las condiciones iniciales. Así mismo, se demostró la unicidad de la solución generalizada.

Palabras claves: Ecuaciones diferenciales hiperbólicas, existencia y unicidad de la solución generalizada.

\section{ABSTRACT}

The purpose of this article was to investigate that with the development of functional analysis and distributions theory it is possible to establish generalized solutions that allow solving hyperbolic equations that model the propagation of a wave in an elastic medium subjected to an external force. From a physical point of view, this hyperbolic model requires that the existence of a solution

be physically acceptable and "controllable". Mathematically this translates into problems of existence, uniqueness and continuous dependence of the initial and boundary conditions. The deductive logic method was used for the development of the article to demonstrate the existence and uniqueness of the generalized solution of the problem of hyperbolic evolution, which consisted in approximating the solution of the problem by linear autofunctions of finite dimension and then constructing a dense subspace in a Hilbert space Separable. Then we divide it into stages: Approximation of approximate solutions, convergence of approximate solutions and verification of initial conditions. Likewise, the uniqueness of the generalized solution was demonstrated.

Keywords: Hyperbolic differential equations, existence and uniqueness of the generalized solution.

\footnotetext{
1 Maestro en Ciencias mención en Matemáticas

Docente de la Facultad de Ciencias - Universidad Nacional Jorge Basadre Grohmann - Tacna Perú

2 Licenciado en Matemáticas

Docente de la Facultad de Ciencias - Universidad Nacional Jorge Basadre Grohmann - Tacna Perú

3 Magister en Microelectrónica

Docente de la Facultad de Ciencias - Universidad Nacional Jorge Basadre Grohmann - Tacna Perú

4 Magister en Docencia Universitaria y Gestión Educativa

Docente de la Facultad de Ciencias - Universidad Nacional Jorge Basadre Grohmann - Tacna Perú
} 


\section{INTRODUCCIÓN}

En el estudio de los fenómenos de la naturaleza surgen dos tipos de ecuaciones diferenciales: las ordinarias $y$ las parciales. Ambas constituyen un amplio mundo matemático de gran valor en el desarrollo de las matemáticas. Pero una ecuación diferencial, por sí sola, no es suficiente para dar información concreta sobre un determinado problema. Al estudiar a la naturaleza es necesario dar condiciones extras, que fundamentalmente, son del tipo: Condiciones iniciales $y / 0$ condiciones de contorno. Así en forma natural surgen los dos problemas básicos de la teoría: El problema de Cauchy y el problema de valor en la frontera. (Ortiz, 1998) ecuación diferencial parcial hiperbólica

Las ecuaciones de evolución son ecuaciones en derivadas parciales, las cuales describen procesos que se desarrollan con el tiempo. En una ecuación de este tipo, el estado inicial del sistema es parte de los datos auxiliares que tiene un problema bien planteado.

Consideremos una ecuación hiperbólica, representado por un modelo matemático referente a la propagación de una onda (acústica, electromagnética, etc.) en un medio elástico homogéneo sometida a una fuerza externa. Para una ecuación de este tipo, las condiciones de Dirichlet expresa que la barra elástica esta fija en el borde de la frontera y el estado inicial del sistema son los datos de Cauchy: La configuración inicial (también llamado desplazamiento inicial) y la velocidad inicial. (Brézis ,1984)

Pero una dificultad presenta la ecuación hiperbólica, como por ejemplo, no se tiene una solución generalizada para la existencia y unicidad. En estos términos, el problema es establecer la existencia de una función solución que satisfaga las condiciones iniciales y de contorno, y verificar que esta solución es única. Determinar la existencia y unicidad de la solución correspondiente a una modelado para la propagación de una onda en un medio elástico homogéneo, es un problema delicado que nos propusimos investigar, ya que no disponíamos hasta ese momento de ningún medio para existencia y unicidad salvo que hallemos efectivamente la solución clásica de la ecuación de evolución hiperbólica, lo que puede resultar difícil sino imposible.

Así, frente a la dificultad, el análisis funcional es una herramienta fundamental en la investigación de los problemas de evolución, más precisamente, espacios funcionales dentro de la teoría de las distribuciones que son usadas para el estudio de los teoremas de existencia y unicidad de la solución generalizada de las ecuaciones en derivadas parciales, las cuales han sido exitosamente usados por muchos matemáticos de gran prestigio como Adams (1975); Brézis (1984); Medeiros (1990)

La investigación es un problema sobre la existencia y unicidad de una solución generalizada de una ecuación diferencial hiperbólica que modela la propagación de una onda en un medio elástico sometida a una fuerza externa, la que se justifica porque permite analizar los problemas provenientes de la física-matemática, modelado por ecuaciones de evolución en el lenguaje de la teoría de las distribuciones y del análisis funcional. Así mismo, permitirá aplicar los métodos y las técnicas de la propagación de una onda al estudiar otras ecuaciones diferenciales, parciales.

La aparición de la función delta de Dirac, conocida desde la época de Fourier, jugó un papel central en el manejo de nuevas ideas, lo que se fortaleció con su uso por parte de Heaviside, y por el trabajo de Dirac en el estudio de la mecánica cuántica. Todo ello, entre otros aspectos, fue consolidado con nuevos aportes, por el matemático francés Laurent Schwartz al idealizar la teoría de las distribuciones (1940); se abre así una nueva era en el estudio de las ecuaciones en derivadas parciales. (Ortiz, 1998)

\section{Teoría de Distribuciones}

Los espacios serán definidos sobre un conjunto abierto $\Omega \subset \mathbb{R}^{n}$.

Definición (Soporte de una función) Sea $\varphi$ una función real de variable vectorial. Se denomina soporte de $\varphi$ al conjunto

$\operatorname{sop} \varphi=\overline{\left\{x \in \mathbb{R}^{\mathrm{n}} / \varphi(\mathrm{x}) \neq 0\right\}}$.

Definición (EspacioC $C_{0}^{\infty}(\Omega)$ ) El espacio $C_{0}^{\infty}(\Omega)$ es el espacio vectorial de las funciones 
infinitamente diferenciables en $\Omega$ y tienen su soporte contenido en un compacto de $\Omega$.

Definición (Convergencia) Una sucesión de funciones $\left(\varphi_{\mathrm{j}}\right)$ converge para para $\varphi$ en $\mathrm{C}_{0}^{\infty}(\Omega)$, si satisface las siguientes condiciones:

a) $\operatorname{sop}\left(\varphi_{j}\right) \subset K$, donde $K$ es un compacto fijo en $\Omega, \forall j=1,2, \ldots$

b) $\left(\varphi_{j}\right)$ converge uniformemente para $\varphi$ en $\Omega$, juntamente con todas sus derivadas de cualquier orden.

Definición (Espacio de las funciones de prueba). El espacio de las funciones de prueba es el espacio vectorial $\mathrm{C}_{0}^{\infty}(\Omega)$ con la convergencia en $\mathrm{C}_{0}^{\infty}(\Omega)$.

Definición (Espacio de una Distribución) Una distribución sobre $\Omega$ es una funcional lineal $\mathrm{T}$, continua en el espacio de las funciones de prueba $\mathrm{D}(\Omega)$.

Definición. (Derivada de una distribución) La derivada de una distribución $T$ sobre un abierto $\Omega$ de $\mathbb{R}^{\mathrm{n}}$ es una funcional lineal $\frac{\partial \mathrm{T}}{\partial \mathrm{x}_{\mathrm{k}}}$, continua en el espacio de las funciones de prueba $D(\Omega)$, definida por la relación

$$
\left\langle\frac{\partial \mathrm{T}}{\partial \mathrm{x}_{\mathrm{k}}}, \varphi\right\rangle=-\left\langle\mathrm{T}, \frac{\partial \varphi}{\partial \mathrm{x}_{\mathrm{k}}}\right\rangle, \forall \varphi \in \mathrm{D}(\Omega), \mathrm{k}=\overline{1, \mathrm{n} .}
$$

\section{Espacios de Sobolev}

Sea $\Omega \subset \mathbb{R}^{n}$ un conjunto abierto y sea $\mathrm{p} \in$ $\mathbb{R} \operatorname{con} 1 \leq \mathrm{p} \leq \infty$.

Definición. El espacio de Sobolev $\mathrm{W}^{1, \mathrm{p}}(\Omega)$ es el conjunto de funciones $u \in \mathrm{L}^{\mathrm{p}}(\Omega)$, tales que todas las derivadas parciales.

$\frac{\partial u}{\partial x_{i}}, i=1,2, \ldots, n$, pertenecen $a L^{p}(\Omega)$, en el sentido de las distribuciones sobre $\Omega$.

El espacio $\mathrm{W}^{1, \mathrm{p}}(\Omega)$ está dotado de la norma

$$
\|u\|_{w^{1, p}}^{p}=\|u\|_{L^{p}}^{p}+\sum_{i=1}^{n}\left|\frac{\partial u}{\partial x_{i}}\right|_{L^{p}}^{p}
$$

El espacio $\mathrm{W}^{1, \mathrm{p}}(\Omega)$ es un espacio de Banach para $1 \leq p \leq \infty ; \mathrm{W}^{1, \mathrm{p}}(\Omega)$ es reflexivo para $1<$ $p<\infty$ y separable para $1 \leq p<\infty$.

Notación. $\mathrm{H}^{1}(\Omega)=\mathrm{W}^{1, \mathrm{p}}(\Omega)$, es el espacio de Sobolev de orden uno.

\section{Definición. (El espacio $\mathrm{W}^{1, p}(\Omega)$ ) Sea}

$\mathrm{p} \in \mathbb{R}$ con $1 \leq \mathrm{p}<\infty$, se define el espacio $\mathrm{W}_{0}^{1, \mathrm{p}}(\Omega)$ como la cerradura de $\mathrm{D}(\Omega)$ en $\mathrm{W}^{1, \mathrm{p}}(\Omega)$ , el cual está dotado de la norma $\|\mathrm{u}\|_{\mathrm{w}_{0}^{1, \mathrm{p}}}^{\mathrm{p}}=$ $\sum_{\mathrm{i}=1}^{\mathrm{n}}\left|\frac{\partial \mathrm{u}}{\partial \mathrm{x}_{\mathrm{i}}}\right|_{\mathrm{L}^{\mathrm{p}}}^{\mathrm{p}}$

El espacio $\mathrm{W}_{0}^{1, \mathrm{p}}(\Omega)$ dotado de la norma inducida por $\mathrm{W}^{1, \mathrm{p}}(\Omega)$ es un espacio de Banach separable; es reflexivo si $1 \leq \mathrm{p}<\infty$

Definición. (El espacioH $\mathbf{H}_{0}^{1}(\Omega)$ ) Se define el espacio $\mathrm{H}_{0}^{1}(\Omega)$ como la cerradura de $\mathrm{D}(\Omega)$ en $\mathrm{H}^{1}(\Omega)$, el cual está dotado con la norma

$$
\|\mathrm{u}\|_{\mathrm{H}_{0}^{1}}^{2}=\sum_{\mathrm{i}=1}^{\mathrm{n}}\left|\frac{\partial \mathrm{u}}{\partial \mathrm{x}_{\mathrm{i}}}\right|_{\mathrm{L}^{2}}^{2}
$$

El espacio $\mathrm{H}_{0}^{1}(\Omega)$ es un espacio de Hilbert separable, con el producto escalar de $\mathrm{H}^{1}(\Omega)$.

Propiedades que serán usados para la existencia y unicidad de la solución generalizada

Teorema de Lax-Milgram. Sea $\mathbf{a}: \mathbf{V x V} \rightarrow \mathbb{R}$ una forma bilineal continua y coerciva en $\mathbf{V}$ y $\mathbf{f} \in$ $\mathbf{V}^{\prime}$. Entonces existe un único $\mathrm{u} \in \mathrm{V}$ tal que $\mathbf{a}(\mathbf{u}, \mathbf{v})=\langle\mathbf{f}, \mathbf{v}\rangle \forall \mathbf{v} \in \mathbf{V}$.

Lema de Gronwall. Sea $\varphi:(0, T) \rightarrow \mathbb{R}$ absolutamente continua tal que $\varphi(t) \geq 0 \quad \forall t \in$ $(0, T)$.Si $\varphi(t) \leq c_{1}+c_{2} \int_{0}^{t} \varphi(s) d s$, entonces $\varphi(\mathrm{t}) \leq \mathrm{c}$.

\section{MATERIAL Y MÉTODO}

En la investigación nuestro material de estudio fue un modelo matemático referente a la propagación de una onda (acústica, electromagnética, etc.) en un medio elástico $\Omega \subset \mathbb{R}^{n}$. Sea $\Omega$ un conjunto abierto, acotado y bien regular de $\mathbb{R}^{n}$, cuya frontera lo denotamos por $\Gamma$. Considere el cilindro $Q=\Omega \times(0, T)$ siendo $0<T<+\infty$. La frontera lateral del cilindro $Q$, será representada por $\Gamma$. Aquí $\Sigma=\Gamma \mathrm{x}(0, \mathrm{~T})$.

El problema de hiperbólico no homogéneo consistió en que dados $\mathrm{u}_{0} \in \mathrm{L}^{2}(\Omega) \mathrm{y}$ $\mathrm{f} \in \mathrm{L}^{2}(\mathrm{Q})$ encontrar una función real $\mathrm{u}=\mathrm{u}(\mathrm{x}, \mathrm{t})$ definida en $Q$, satisfaciendo las siguientes condiciones 
(1.1) $\left\{\begin{array}{c}\frac{\partial^{2} \mathrm{u}}{\partial \mathrm{t}^{2}}-\Delta \mathrm{u}=\mathrm{f} \text { en } \mathrm{Q} \\ \mathrm{u}=0 \quad \text { sobre } \Sigma \\ \mathrm{u}(\mathrm{x}, 0)=\mathrm{u}_{0}(\mathrm{x}) \text { en } \Omega \\ \frac{\partial \mathrm{u}(\mathrm{x}, 0)}{\partial \mathrm{t}}=\mathrm{u}_{1}(\mathrm{x}) \text { en } \Omega\end{array}\right.$

, donde $\Delta=\sum_{i=1}^{n} \frac{\partial^{2}}{\partial x_{i}^{2}} \quad$ designa el Laplaciano respecto de las variables espaciales, $t$ es la variable tiempo, $\mathrm{u}_{0} \mathrm{y} \mathrm{u}_{1}$ son funciones dadas.

Se empleó en el desarrollo del artículo el método lógico deductivo, para demostrar la existencia y unicidad de la solución generalizada para el problema de evolución hiperbólica; que consistió en aproximar la solución del problema por autofunciones lineales de dimensión finita, para luego construir un subespacio denso en un espacio de Hilbert separable.

\section{RESULTADOS}

\section{Existencia de la solución generalizada de la propagación de una onda}

Acotación de las soluciones aproximadas:

Si $v=u_{m}^{\prime}(t)$ en la solución del problema aproximado $\frac{d}{d t}\left(u_{m}^{\prime}(t), u_{m}^{\prime}(t)\right)+$ $a\left(u_{m}(t), u_{m}^{\prime}(t)\right)=\left(f(t), u_{m}^{\prime}(t)\right)$, entonces $\left|\mathrm{u}_{\mathrm{m}}^{\prime}(\mathrm{t})\right|_{\mathrm{L}^{2}(\Omega)} \leq \mathrm{c}$.

Convergencia de las soluciones aproximadas: Existen subsucesiones de $\left(u_{m}^{\prime}\right)$ y $\left(u_{m}\right)$ tales que $u_{m}^{\prime} \rightarrow u^{\prime}$ débil estrella en $L^{\infty}\left(0, T ; L^{2}(\Omega)\right), y$ $\mathrm{u}_{\mathrm{m}} \rightarrow \mathrm{u}$ débil estrella en $\mathrm{L}^{\infty}\left(0, \mathrm{~T} ; \mathrm{H}_{0}^{1}(\Omega)\right)$, entonces

$\frac{\mathrm{d}}{\mathrm{dt}}\left(\mathrm{u}^{\prime}(\mathrm{t}), \mathrm{v}\right)+\mathrm{a}(\mathrm{u}(\mathrm{t}), \mathrm{v})=(\mathrm{f}(\mathrm{t}), \mathrm{v})$

$\forall \mathrm{v} \in \mathrm{H}_{0}^{1}(\Omega)$ en $\mathrm{D}^{\prime}(0, \mathrm{~T})$.

Verificación de las condiciones

iniciales:i) $\left.u(0)=u_{0} i i\right) u_{1}=u^{\prime}(0)$

\section{Unicidad de la solución generalizada de la} onda

Sean $\tilde{\mathrm{u}}_{1}(\mathrm{t})$ y $\tilde{\mathrm{u}}_{2}(\mathrm{t})$ dos soluciones que satisfacen la ecuación

$-\left(u_{1}, \mathrm{v}\right)-\int_{0}^{\mathrm{T}}\left(\mathrm{u}^{\prime}(t), \mathrm{v}\right) \theta^{\prime}(\mathrm{t}) \mathrm{dt}+$ $\int_{0}^{\mathrm{T}} \mathrm{a}\left(\mathrm{u}_{\mathrm{m}}(\mathrm{t}), \mathrm{v}\right) \theta(\mathrm{t}) \mathrm{dt}=\int_{0}^{\mathrm{T}}(\mathrm{f}(\mathrm{t}), \mathrm{v}) \theta(\mathrm{t}) \mathrm{dt}$, entonces $\tilde{\mathrm{u}}_{1}(\mathrm{t})=\tilde{\mathrm{u}}_{2}(\mathrm{t})$

\section{DISCUSIÓN}

Existencia y unicidad de la solución generalizada de la onda

Teorema. Sean $\mathrm{f} \in \mathrm{L}^{2}(\mathrm{Q}), \mathrm{u}_{0} \in \mathrm{H}_{0}^{1}(\Omega), \mathrm{u}_{1} \in$ $\mathrm{L}^{2}(\Omega)$. Entonces existe una única u: $[0, \mathrm{~T}] \rightarrow \mathrm{H}_{0}^{1}$ solución generalizada de (1.1) que satisface:

$$
\begin{gathered}
\frac{\mathrm{d}}{\mathrm{dt}}\left(\mathrm{u}^{\prime}(\mathrm{t}), \mathrm{v}\right)+\mathrm{a}(\mathrm{u}(\mathrm{t}), \mathrm{v})=(\mathrm{f}(\mathrm{t}), \mathrm{v}) \\
\forall \mathrm{v} \in \mathrm{H}_{0}^{1}(\Omega) \text { en el sentido } \mathrm{D}^{\prime}(0, \mathrm{~T})
\end{gathered}
$$

$\mathrm{u} \in \mathrm{L}^{\infty}\left(0, \mathrm{~T} ; \mathrm{H}_{0}^{1}(\Omega)\right)$

$\mathrm{u}^{\prime} \in \mathrm{L}^{\infty}\left(0, \mathrm{~T} ; \mathrm{L}^{2}(\Omega)\right)$

$\mathrm{u}(0)=\mathrm{u}_{0}$

$\mathrm{u}^{\prime}(0)=\mathrm{u}_{1}$

\section{Demostración}

El espacio $H_{0}^{1}(\Omega)$ es un espacio de Hilbert separable, entonces existe $\mathrm{w}_{1}, \mathrm{w}_{2}, \ldots, \mathrm{w}_{\mathrm{m}}$ una base ortonormal completo en $\mathrm{H}_{0}^{1}(\Omega)$. El espacio $V_{m}=\left[w_{1}, w_{2}, \ldots, w_{m}\right]$ es un espacio denso en $\mathrm{H}_{0}^{1}(\Omega)$.

Considere el problema aproximado:

$$
\frac{\mathrm{d}}{\mathrm{dt}}\left(\mathrm{u}_{\mathrm{m}}^{\prime}(\mathrm{t}), \mathrm{v}\right)+\mathrm{a}\left(\mathrm{u}_{\mathrm{m}}(\mathrm{t}), \mathrm{v}\right)=
$$

$(\mathrm{f}(\mathrm{t}), \mathrm{v}) \quad \forall \mathrm{v} \in V_{m}$

$\mathrm{u}_{\mathrm{m}}(0)=\mathrm{u}_{0 \mathrm{~m}}, \mathrm{u}_{0 \mathrm{~m}}=\sum_{v=1}^{\mathrm{m}} \alpha_{0 v} \mathrm{w}_{v} \rightarrow \mathrm{u}_{0}$ $\mathrm{enH}_{0}^{1}(\Omega)$

$\mathrm{u}_{\mathrm{m}}^{\prime}(0)=\mathrm{u}_{1 \mathrm{~m}}, \mathrm{u}_{1 \mathrm{~m}}=\sum_{v=1}^{\mathrm{m}} \beta_{1 v} \mathrm{w}_{v} \rightarrow \mathrm{u}_{1} \quad$ en $\mathrm{L}^{2}(\Omega)$.

La demostración se divide en etapas:

\section{Etapa 1. Acotación de las soluciones} aproximadas:

Sea $v=u_{m}^{\prime}(t), y$ sustituyendo en (1) se obtiene

(2) $\frac{d}{d t}\left(u_{m}^{\prime}(t), u_{m}^{\prime}(t)\right)+a\left(u_{m}(t), u_{m}^{\prime}(t)\right)=$ $\left(\mathrm{f}(\mathrm{t}), \mathrm{u}_{\mathrm{m}}^{\prime}(\mathrm{t})\right)$.

Aplicando propiedades a (2) tenemos $\frac{\mathrm{d}\left|\mathrm{u}_{\mathrm{m}}^{\prime}(\mathrm{t})\right|_{\mathrm{L}^{2}(\Omega)}^{2}}{\mathrm{dt}} \quad+\frac{1}{2} \frac{\mathrm{da}\left(\mathrm{u}_{\mathrm{m}}(\mathrm{t}), \mathrm{u}_{\mathrm{m}}(\mathrm{t})\right.}{\mathrm{dt}}=\left(\mathrm{f}(\mathrm{t}), \mathrm{u}_{\mathrm{m}}^{\prime}(\mathrm{t})\right)$ (3) $\frac{1}{2} \frac{\mathrm{d}}{\mathrm{dt}}\left[2\left|\mathrm{u}_{\mathrm{m}}^{\prime}(\mathrm{t})\right|_{\mathrm{L}^{2}(\Omega)}^{2}+\mathrm{a}\left(\mathrm{u}_{\mathrm{m}}(\mathrm{t}), \mathrm{u}_{\mathrm{m}}(\mathrm{t})\right)\right]=$ $\left(\mathrm{f}(\mathrm{t}), \mathrm{u}_{\mathrm{m}}^{\prime}(\mathrm{t})\right)$.

Integrando (3) sobre $[0, \mathrm{t}]$ resulta $(4) \frac{1}{2}\left[2\left|u_{m}^{\prime}(t)\right|_{L^{2}(\Omega)}^{2}+a\left(u_{m}(t), u_{m}(t)\right)\right]=$ $\frac{1}{2}\left[2\left|u_{m}^{\prime}(0)\right|_{L^{2}(\Omega)}^{2}+a\left(u_{m}(0), u_{m}(0)\right)\right]+$ $\int_{0}^{\mathrm{t}}\left(\mathrm{f}(\mathrm{t}), \mathrm{u}_{\mathrm{m}}^{\prime}(\mathrm{t})\right) \mathrm{dt}$. 
Aplicando en (4) la propiedad en la forma bilineal que $a$ es coerciva, se obtiene

$$
\begin{gathered}
\left.\frac{1}{2}\left[2\left|\mathrm{u}_{\mathrm{m}}^{\prime}(\mathrm{t})\right|_{\mathrm{L}^{2}(\Omega)}^{2}+\alpha\left\|\mathrm{u}_{\mathrm{m}}(\mathrm{t})\right\|_{\mathrm{H}^{1}(\Omega)}^{2}\right)\right] \leq \\
\leq \int_{0}^{\mathrm{t}}\left(\mathrm{f}(\mathrm{t}), \mathrm{u}_{\mathrm{m}}^{\prime}(\mathrm{t})\right) \mathrm{dt} \leq \mathrm{c} \\
\quad+\int_{0}^{\mathrm{t}}|\mathrm{f}(\mathrm{s})|_{\mathrm{L}^{2}(\Omega)}\left|\mathrm{u}_{\mathrm{m}}^{\prime}(\mathrm{s})\right|_{\mathrm{L}^{2}(\Omega)} \mathrm{ds} \leq \\
\leq \mathrm{c}+\frac{1}{2} \int_{0}^{\mathrm{t}}|\mathrm{f}(\mathrm{s})|_{\mathrm{L}^{2}(\Omega)}^{2} \mathrm{ds}+\frac{1}{2} \int_{0}^{\mathrm{t}}\left|\mathrm{u}_{\mathrm{m}}^{\prime}(\mathrm{s})\right|_{\mathrm{L}^{2}(\Omega)}^{2} \mathrm{ds} \\
\leq \mathrm{c}+\frac{1}{2} \int_{0}^{\mathrm{T}}|\mathrm{f}(\mathrm{s})|_{\mathrm{L}^{2}(\Omega)}^{2} \mathrm{ds}+\frac{1}{2} \int_{0}^{\mathrm{t}}\left|\mathrm{u}_{\mathrm{m}}^{\prime}(\mathrm{s})\right|_{\mathrm{L}^{2}(\Omega)}^{2} \mathrm{ds}= \\
=\mathrm{c}_{1}+\frac{1}{2} \int_{0}^{\mathrm{t}}\left|\mathrm{u}_{\mathrm{m}}^{\prime}(\mathrm{s})\right|_{\mathrm{L}^{2}(\Omega)}^{2} \mathrm{ds}
\end{gathered}
$$

Por consiguiente

$(5)\left|u_{m}^{\prime}(t)\right|_{L^{2}(\Omega)}^{2} \leq c_{1}+\frac{1}{2} \int_{0}^{t}\left|u_{m}^{\prime}(s)\right|_{L^{2}(\Omega)}^{2} d s=c_{1}+$ $c_{2} \int_{0}^{t}\left|u_{m}^{\prime}(s)\right|_{L^{2}(\Omega)}^{2} d s$

Aplicando Gronwall en (5) tenemos

$\left|u_{m}^{\prime}(t)\right|_{L^{2}(\Omega)}^{2}$, es acotada e independientemente de $t$ y $m$, es decir,

$\left|u_{m}^{\prime}(t)\right|_{L^{2}(\Omega)} \leq \mathrm{c}$.

Conclusión:

$\left(u_{m}^{\prime}\right)$ es acotado en el espacio $L^{\infty}\left(0, T ; L^{2}(\Omega)\right)$

$\left(u_{m}\right)$ es acotado en el espacio $L^{\infty}\left(0, T ; H_{0}^{1}(\Omega)\right)$

\section{Etapa 2. Convergencia de las soluciones aproximadas:}

Sean las subsucesiones de

$\left(u_{m}^{\prime}\right)$ y $\left(u_{m}\right)$ tales que

(6) $u_{m}^{\prime} \rightarrow u^{\prime}$ débil estrella en $L^{\infty}\left(0, T ; L^{2}(\Omega)\right)$

(7) $\mathrm{u}_{\mathrm{m}} \rightarrow \mathrm{u}$ débil estrella en $\mathrm{L}^{\infty}\left(0, \mathrm{~T} ; \mathrm{H}_{0}^{1}(\Omega)\right)$ Por

el estudio de $\mathrm{L}^{1}$ en (6) y (7) se obtienen

(8) $\int_{0}^{\mathrm{T}}\left(\mathrm{u}_{\mathrm{m}}^{\prime}(\mathrm{t}), \mathrm{w}(\mathrm{t})\right) \mathrm{dt} \rightarrow \int_{0}^{\mathrm{T}}\left(\mathrm{u}^{\prime}(\mathrm{t}), \mathrm{w}(\mathrm{t})\right) d \mathrm{t}$

$\forall \mathrm{w} \in \mathrm{L}^{1}\left(0, \mathrm{~T} ; \mathrm{L}^{2}(\Omega)\right), \mathrm{y}$

(9) $\int_{0}^{\mathrm{T}}\left(\left(\mathrm{u}_{\mathrm{m}}(\mathrm{t}), \mathrm{w}(\mathrm{t})\right)\right) \mathrm{dt} \rightarrow \int_{0}^{\mathrm{T}}((\mathrm{u}(\mathrm{t}), \mathrm{w}(\mathrm{t}))) \mathrm{dt}$

$\forall \mathrm{w} \in \mathrm{L}^{1}\left(0, \mathrm{~T} ; \mathrm{H}_{0}^{1}(\Omega)\right)$.

En la ecuación aproximada (1) se tiene

$\mathrm{v} \in \mathrm{V}_{\mathrm{m}}$ en $\mathrm{D}^{\prime}(0, \mathrm{~T})$, en particular sea $\mathrm{v}=\theta(\mathrm{t}) \mathrm{z}$

tal que $\mathrm{z} \in \mathrm{L}^{2}(\Omega)$ y $\theta \in \mathrm{D}(0, \mathrm{~T})$.

Entonces, integrando se obtiene

$\frac{\mathrm{d}}{\mathrm{dt}} \int_{\Omega} \mathrm{u}_{\mathrm{m}}^{\prime}(\mathrm{t}) \theta(\mathrm{t}) \mathrm{zdx}+\mathrm{a}\left(\mathrm{u}_{\mathrm{m}}(\mathrm{t}), \theta(\mathrm{t}) \mathrm{z}\right)$

$$
=\int_{\Omega} f(t) \theta(t) z d x
$$

$\frac{d}{d t} \int_{\Omega} \int_{0}^{T} u_{m}^{\prime}(t) \theta(t) z d t d x+\int_{0}^{T} a\left(u_{m}(t), \theta(t) z\right) d t=$ $\int_{\Omega} \int_{0}^{\mathrm{T}} \mathrm{f}(\mathrm{t}) \theta(\mathrm{t}) \mathrm{zdtdx}$
(10) $\int_{\Omega} \int_{0}^{T} u_{m}^{\prime}(t) \theta^{\prime}(t) z d t d x+$ $\int_{0}^{\mathrm{T}} \mathrm{a}\left(\mathrm{u}_{\mathrm{m}}(\mathrm{t}), \theta(\mathrm{t}) \mathrm{z}\right) d \mathrm{t}=\int_{\Omega} \int_{0}^{\mathrm{T}} \mathrm{f}(\mathrm{t}) \theta(\mathrm{t}) \mathrm{zdtdx}$.

Sea $w \in L^{1}\left(0, T ; L^{2}(\Omega)\right)$ tal que $w(t)=$ $\theta^{\prime}(\mathrm{t}) \mathrm{z}, \mathrm{z} \in \mathrm{L}^{2}(\Omega)$ y $\theta^{\prime}(\mathrm{t}) \in \mathrm{D}(0, \mathrm{~T})$

Por la parte (6) tenemos

$$
\begin{aligned}
&-\int_{\Omega} \int_{0}^{T} u_{m}^{\prime}(t) \theta^{\prime}(t) z d t d x \\
& \rightarrow-\int_{\Omega} \int_{0}^{T} u^{\prime}(t) \theta^{\prime}(t) z d t d x
\end{aligned}
$$

Por otro lado se tiene

(11) $\quad a\left(u_{m}(t), \theta(t) z\right)=\left(\left(u_{m}(t), \theta(t) z\right)\right)$ en el espacio $\mathrm{H}_{0}^{1}(\Omega)$, y para todo $\mathrm{w} \in \mathrm{L}^{1}\left(0, \mathrm{~T} ; \mathrm{H}^{-1}(\Omega)\right)$ tal que $\mathrm{w}(\mathrm{t})=\theta(\mathrm{t}) \mathrm{z}$.

Entonces se tiene

$$
\int_{0}^{\mathrm{T}} \mathrm{a}\left(\mathrm{u}_{\mathrm{m}}(\mathrm{t}), \theta(\mathrm{t}) \mathrm{z}\right) \mathrm{dt}=\int_{0}^{\mathrm{T}}\left(\left(\mathrm{u}_{\mathrm{m}}(\mathrm{t}), \theta(\mathrm{t}) \mathrm{z}\right)\right) \mathrm{dt}
$$

Por la parte (7) tenemos

$$
\int_{0}^{\mathrm{T}}\left(\left(\mathrm{u}_{\mathrm{m}}(\mathrm{t}), \theta(\mathrm{t}) \mathrm{z}\right)\right) \mathrm{dt} \rightarrow
$$

$\int_{0}^{\mathrm{T}}((\mathrm{u}(\mathrm{t}), \theta(\mathrm{t}) \mathrm{z})) \mathrm{dt}=\int_{0}^{\mathrm{T}} \mathrm{a}(\mathrm{u}(\mathrm{t}), \theta(\mathrm{t}) \mathrm{z}) \mathrm{dt}$,

$Y$ reemplazando en (10) se obtiene

$-\int_{\Omega} \int_{0}^{\mathrm{T}} \mathrm{u}^{\prime}(\mathrm{t}) \theta^{\prime}(\mathrm{t}) \mathrm{zdtdx}+\int_{0}^{\mathrm{T}} \mathrm{a}(\mathrm{u}(\mathrm{t}), \theta(\mathrm{t}) \mathrm{z}) \mathrm{dt}=$

$$
=\int_{\Omega} \int_{0}^{T} f(t) \theta(t) z d t d x
$$

$\frac{\mathrm{d}}{\mathrm{dt}} \int_{\Omega} \int_{0}^{\mathrm{T}} \mathrm{u}^{\prime}(\mathrm{t}) \theta(\mathrm{t}) \mathrm{zdtdx}+\int_{0}^{\mathrm{T}} \mathrm{a}(\mathrm{u}(\mathrm{t}), \theta(\mathrm{t}) \mathrm{z}) \mathrm{dt}=$

$$
=\int_{\Omega} \int_{0}^{T} f(t) \theta(t) z d t d x
$$

$$
\frac{d}{d t} \int_{\Omega} u^{\prime}(t) \theta(t) z d x+a(u(t), \theta(t) z)=
$$

$\int_{\Omega} \mathrm{f}(\mathrm{t}) \theta(\mathrm{t}) \mathrm{zdx}$

Se sabe que $v=\theta(t) z$ y reemplazando en (12) resulta que

(13) $\frac{\mathrm{d}}{\mathrm{dt}}\left(\mathrm{u}^{\prime}(\mathrm{t}), \mathrm{v}\right)+\mathrm{a}(\mathrm{u}(\mathrm{t}), \mathrm{v})=(\mathrm{f}(\mathrm{t}), \mathrm{v}) \forall \mathrm{v} \in$

$\mathrm{H}_{0}^{1}(\Omega)$

En el sentidoD ${ }^{\prime}(0, T)$.

\section{Etapa 3. Verificación de las condiciones iniciales de la solución generalizada}

Primero se demuestra que $u(0)=u_{0}$

De (6) sabemos que

$u_{m} \rightarrow u$ débil estrella en $L^{\infty}\left(0, T ; H_{0}^{1}(\Omega)\right)$

, y al integrar sobre $[0, \mathrm{~T}]$ se obtiene

(14) $\int_{0}^{\mathrm{T}}\left(\mathrm{u}_{\mathrm{m}}(\mathrm{t}), \mathrm{w}(\mathrm{t})\right) \mathrm{dt} \rightarrow \int_{0}^{\mathrm{T}}(\mathrm{u}(\mathrm{t}), \mathrm{w}(\mathrm{t})) \mathrm{dt}$

$\forall \mathrm{w} \in \mathrm{L}^{1}\left(0, \mathrm{~T} ; \mathrm{H}_{0}^{1}(\Omega)\right)$.

Sea $\mathrm{w}(\mathrm{t})=\dot{\theta}(\mathrm{t}) \mathrm{z}, \mathrm{z} \in \mathrm{H}_{0}^{1}(\Omega)$ tal que $\theta(0)=$ $1 ; \theta(\mathrm{T})=0$. En (14) se obtiene 
$(15) \int_{0}^{\mathrm{T}}\left(u_{m}(t), z\right) \dot{\theta}(\mathrm{t}) \mathrm{dt} \rightarrow \int_{0}^{\mathrm{T}}(u(t), z) \dot{\theta}(\mathrm{t}) \mathrm{dt}$

Integrando por partes en (15) se obtiene (16)

$\int_{0}^{\mathrm{T}}\left(\mathrm{u}_{\mathrm{m}}(\mathrm{t}), \mathrm{z}\right) \dot{\theta}(\mathrm{t}) d \mathrm{t}=-\left(\mathrm{u}_{\mathrm{m}}(0), \mathrm{z}\right)-$

$\int_{0}^{T} \frac{d}{d t}\left(u_{m}(t), z\right) \theta(t) d t$.

Aplicando convergencia en (16) resulta

(17) $\int_{0}^{\mathrm{T}}(\mathrm{u}(\mathrm{t}), \mathrm{z}) \dot{\theta}(\mathrm{t}) \mathrm{dt}=-\left(\mathrm{u}_{0}, \mathrm{z}\right)-$

$\int_{0}^{\mathrm{T}} \frac{\mathrm{d}}{\mathrm{dt}}(\mathrm{u}(\mathrm{t}), \mathrm{z}) \theta(\mathrm{t}) \mathrm{dt}$.

Integrando por partes en (14) se obtiene

(18) $\quad \int_{0}^{\mathrm{T}}(\mathrm{u}(\mathrm{t}), \mathrm{z}) \dot{\theta}(\mathrm{t}) \mathrm{dt}=-(\mathrm{u}(0), \mathrm{z})-$ $\int_{0}^{\mathrm{T}} \frac{\mathrm{d}}{\mathrm{dt}}(\mathrm{u}(\mathrm{t}), \mathrm{z}) \theta(\mathrm{t}) \mathrm{dt}$

Por la unicidad de límite en (17) y (18) se obtiene

$\left(u_{0}, \mathrm{z}\right)=(u(0), \mathrm{z}) \forall z \in \mathrm{H}_{0}^{1}(\Omega)$,

$Y$ en particular para $z \in \mathrm{D}(\Omega)$ resulta que

$$
u_{0}=u(0)
$$

Segundo se demuestra queu $u_{1}=u^{\prime}(0)$

En (1) multiplicando por $\theta \in \mathrm{C}^{1}([0, \mathrm{~T}] ; \mathbb{R})$ e integrando de $\left[\begin{array}{ll}0, & \mathrm{~T}\end{array}\right]$ se obtiene (19) $\int_{0}^{T} \frac{d}{d t}\left(u_{m}^{\prime}(t), v\right) \theta(t) d t+$ $\int_{0}^{T} \mathrm{a}\left(u_{m}(\mathrm{t}), \mathrm{v}\right) \theta(\mathrm{t}) \mathrm{dt}=\int_{0}^{T}(\mathrm{f}(\mathrm{t}), \mathrm{v}) \theta(\mathrm{t}) \mathrm{dt}$

En (19) tenemos

$\int_{0}^{T} \frac{d}{d t}\left(u_{m}^{\prime}(t), v\right) \theta(t) d t==\left.\left(u_{m}^{\prime}(t), v\right) \theta(t)\right|_{0} ^{T}-$

$\int_{0}^{\mathrm{T}}\left(\mathrm{u}_{\mathrm{m}}^{\prime}(\mathrm{t}), \mathrm{v}\right) \theta^{\prime}(\mathrm{t}) \mathrm{dt}$,

$Y$ resulta que $\int_{0}^{\mathrm{T}} \frac{\mathrm{d}}{\mathrm{dt}}\left(\mathrm{u}_{\mathrm{m}}^{\prime}(\mathrm{t}), \mathrm{v}\right) \theta(\mathrm{t}) \mathrm{dt}=$ $-\left(u_{m}^{\prime}(0), v\right)-\int_{0}^{T}\left(u_{m}^{\prime}(t), v\right) \theta^{\prime}(t) d t$

Reemplazando en (18) se obtiene

(20) $-\left(\mathrm{u}_{\mathrm{m}}^{\prime}(0), \mathrm{v}\right)-\int_{0}^{\mathrm{T}}\left(\mathrm{u}_{\mathrm{m}}^{\prime}(\mathrm{t}), \mathrm{v}\right) \theta^{\prime}(\mathrm{t}) \mathrm{dt}+$ $+\int_{0}^{\mathrm{T}} \mathrm{a}\left(\mathrm{u}_{\mathrm{m}}(\mathrm{t}), \mathrm{v}\right) \theta(\mathrm{t}) \mathrm{dt}=\int_{0}^{\mathrm{T}}(\mathrm{f}(\mathrm{t}), \mathrm{v}) \theta(\mathrm{t}) \mathrm{dt}$

Aplicando la convergencia a (20) se obtiene

(21) $-\left(u_{1}, \mathrm{v}\right)-\int_{0}^{\mathrm{T}}\left(\mathrm{u}^{\prime}(t), \mathrm{v}\right) \theta^{\prime}(\mathrm{t}) \mathrm{dt}+$ $\int_{0}^{T} \mathrm{a}\left(\mathrm{u}_{\mathrm{m}}(\mathrm{t}), \mathrm{v}\right) \theta(\mathrm{t}) d \mathrm{t}=\int_{0}^{\mathrm{T}}(\mathrm{f}(\mathrm{t}), \mathrm{v}) \theta(\mathrm{t}) d \mathrm{t}$

Por otro lado de (13) tenemos que

$\frac{\mathrm{d}}{\mathrm{dt}}\left(\mathrm{u}^{\prime}(\mathrm{t}), \mathrm{v}\right)+\mathrm{a}(\mathrm{u}(\mathrm{t}), \mathrm{v})=(\mathrm{f}(\mathrm{t}), \mathrm{v}) \forall \mathrm{v} \in \mathrm{H}_{0}^{1}(\Omega)$ en el sentido $\mathrm{D}^{\prime}(0, \mathrm{~T})$.

Integrando de $[0, T]$ la ecuación anterior se tiene

(22) $\int_{0}^{T} \frac{d}{d t}\left(u^{\prime}(t), v\right) \theta(t) d t$

$$
\begin{aligned}
& +\int_{0}^{\mathrm{T}} \mathrm{a}(\mathrm{u}(\mathrm{t}), \mathrm{v}) \theta(\mathrm{t}) \mathrm{dt}= \\
& =\int_{0}^{\mathrm{T}}(\mathrm{f}(\mathrm{t}), \mathrm{v}) \theta(\mathrm{t}) \mathrm{dt}
\end{aligned}
$$

$\forall \mathrm{v} \in \mathrm{H}_{0}^{1}(\Omega)$ en $\mathrm{D}^{\prime}(0, \mathrm{~T})$, y resulta

(23) $\int_{0}^{T} \frac{d}{d t}\left(u^{\prime}(t), v\right) \theta(t) d t=-\left(u^{\prime}(0), v\right)-$ $\int_{0}^{\mathrm{T}}\left(\mathrm{u}^{\prime}(\mathrm{t}), \mathrm{v}\right) \theta^{\prime}(\mathrm{t}) \mathrm{dt}$.

Reemplazando en (23) en (22) se obtiene

(24) $-\left(u^{\prime}(0), \mathrm{v}\right)-\int_{0}^{\mathrm{T}}\left(\mathrm{u}^{\prime}(t), \mathrm{v}\right) \theta^{\prime}(\mathrm{t}) \mathrm{dt}+$ $+\int_{0}^{\mathrm{T}} \mathrm{a}(\mathrm{u}(\mathrm{t}), \mathrm{v}) \theta(\mathrm{t}) \mathrm{dt}=\int_{0}^{\mathrm{T}}(\mathrm{f}(\mathrm{t}), \mathrm{v}) \theta(\mathrm{t}) \mathrm{dt}$

Comparando (21) y (24) se tiene que $\left(\mathrm{u}_{1}, \mathrm{v}\right)=$ $\left(\mathrm{u}^{\prime}(0), \mathrm{v}\right) \forall \mathrm{v} \in \mathrm{H}_{0}^{1}(\Omega)$, en particular $\forall \mathrm{v} \in$ $\mathrm{D}(\Omega)$ resulta $u_{1}=u^{\prime}(0)$

\section{Etapa 4. Unicidad de la solución generalizada}

Sean $\mathrm{u}_{1}, \mathrm{u}_{2}$ dos soluciones satisfaciendo (21) tal que

$(25) \mathrm{w}(\mathrm{t})=\tilde{\mathrm{u}}_{1}(\mathrm{t})-\tilde{\mathrm{u}}_{2}(\mathrm{t})$.

Calculando el valor cuando $t=0$ en (25) se tiene $\mathrm{w}(0)=\tilde{\mathrm{u}}_{1}(0)-\tilde{\mathrm{u}}_{2}(0)=u_{0}-u_{0}=0$.

Haciendo $\mathrm{u}^{\prime}(\mathrm{t})=\tilde{\mathrm{u}}_{1}{ }^{\prime}(\mathrm{t})$ y $\mathrm{u}^{\prime}(\mathrm{t})=\tilde{\mathrm{u}}_{2}{ }^{\prime}(\mathrm{t})$ en $(21)$ se tiene

$(26)-\left(\mathrm{u}_{1}, \mathrm{v}\right)-\int_{0}^{\mathrm{T}}\left(\tilde{\mathrm{u}}_{1}{ }^{\prime}(\mathrm{t}), \mathrm{v}\right) \theta^{\prime}(\mathrm{t}) \mathrm{dt}+$ $+\int_{0}^{\mathrm{T}} \mathrm{a}\left(\tilde{\mathrm{u}}_{1}{ }^{\prime}(\mathrm{t}), \mathrm{v}\right) \theta(\mathrm{t}) \mathrm{dt}=\int_{0}^{\mathrm{T}}(\mathrm{f}(\mathrm{t}), \mathrm{v}) \theta(\mathrm{t}) \mathrm{dt}$

$(27)-\left(\mathrm{u}_{1}, \mathrm{v}\right)-\int_{0}^{\mathrm{T}}\left(\tilde{\mathrm{u}}_{2}{ }^{\prime}(\mathrm{t}), \mathrm{v}\right) \theta^{\prime}(\mathrm{t}) \mathrm{dt}+$ $+\int_{0}^{\mathrm{T}} \mathrm{a}\left(\tilde{\mathrm{u}}_{2}{ }^{\prime}(\mathrm{t}), \mathrm{v}\right) \theta(\mathrm{t}) \mathrm{dt}=\int_{0}^{\mathrm{T}}(\mathrm{f}(\mathrm{t}), \mathrm{v}) \theta(\mathrm{t}) \mathrm{dt}$ Restando (26) de (27) se obtiene $-\int_{0}^{\mathrm{T}}\left(\mathrm{w}^{\prime}(\mathrm{t}), \mathrm{v} \theta^{\prime}(\mathrm{t})\right) \mathrm{dt}+$ $\int_{0}^{\mathrm{T}} \mathrm{a}(\mathrm{w}(\mathrm{t}), \mathrm{v} \theta(\mathrm{t})) \mathrm{dt}=0$

Pero $\widetilde{w}(t)=v \theta(t)$, entonces en (28) tenemos (29)

$-\int_{0}^{t}\left(w^{\prime}(t),(v \theta(t))^{\prime}\right) d t+\int_{0}^{t} a(w(t), v \theta(t)) d t=$ 0 
$-\int_{0}^{t}\left(w^{\prime}(t), \widetilde{\mathrm{w}}^{\prime}(\mathrm{t})\right) d t+\int_{0}^{t} a(w(t), \widetilde{\mathrm{w}}(\mathrm{t})) d t=0$ $\forall \widetilde{\mathrm{w}}(\mathrm{t}) \in \mathrm{L}^{1}\left(0, \mathrm{~T} ; \mathrm{H}_{0}^{1}(\Omega)\right)$.

Si escogemos $\widetilde{w}(\mathrm{t})=\left\{\begin{array}{c}\int_{t}^{s} w(t) d t, t \leq s \\ 0, t>s\end{array}\right.$, entonces $\widetilde{w}^{\prime}(\mathrm{t})=w(t)$.

Entonces sustituyéndolo en (30) se obtiene

$$
\begin{aligned}
-\int_{0}^{\mathrm{t}}\left(\mathrm{w}^{\prime}(\mathrm{t}), \widetilde{\mathrm{w}}(\mathrm{t})\right) \mathrm{dt}+\int_{0}^{\mathrm{t}} \mathrm{a}(\mathrm{w}(\mathrm{t}), \widetilde{\mathrm{w}}(\mathrm{t})) \mathrm{dt} & =0 \\
\frac{-1}{2} \int_{0}^{\mathrm{t}} \frac{\mathrm{d}}{\mathrm{dt}}|\mathrm{w}(\mathrm{t})|_{\mathrm{L}^{2}(\Omega)}^{2} \mathrm{dt}+\int_{0}^{\mathrm{t}} \mathrm{a}(\mathrm{w}(\mathrm{t}), \widetilde{\mathrm{w}}(\mathrm{t})) \mathrm{dt} & =0 \\
\frac{-1}{2} \int_{0}^{t} \frac{d}{d t}|w(t)|_{L^{2}(\Omega)}^{2} d t & \\
+\frac{1}{2} \int_{0}^{t} \frac{d}{d t} a(w(t), \mathrm{w}(\mathrm{t})) d t & =0
\end{aligned}
$$

(31) $\frac{-1}{2}\left[|w(t)|_{L^{2}(\Omega)}^{2}-|w(0)|_{L^{2}(\Omega)}^{2}\right]+$

$\frac{1}{2}[a(\widetilde{\mathrm{w}}(\mathrm{t}), \widetilde{\mathrm{w}}(\mathrm{t}))-a(\widetilde{\mathrm{w}}(0), \widetilde{\mathrm{w}}(0))]=0$

Se sabe que $\quad w(0)=0 y \widetilde{w}(t)=0$ entonces

$a(\widetilde{\mathrm{w}}(\mathrm{t}), \widetilde{\mathrm{w}}(\mathrm{t}))=\sum_{i} \int_{\Omega} \frac{\partial \widetilde{\mathrm{w}}(\mathrm{t})}{\partial x} \frac{\partial \widetilde{\mathrm{w}}(\mathrm{t})}{\partial x_{i}} d t=0, \mathrm{de}$

donde resulta $a(\widetilde{\mathrm{w}}(0), \widetilde{\mathrm{w}}(0))=0$

Reemplazando en (31) se tiene que

$-\frac{1}{2}|w(t)|_{L^{2}(\Omega)}^{2}=0$ entonces $\mathrm{w}(\mathrm{t})=0$ para

$0 \leq \mathrm{t} \leq \mathrm{s}$. Se concluye $\tilde{\mathrm{u}}_{1}(\mathrm{t})=\tilde{\mathrm{u}}_{2}(\mathrm{t}) \forall 0 \leq \mathrm{t} \leq$

\section{CONCLUSIONES}

Existe una solución débil para el problema hiperbólico, es decir una función $\mathrm{u}:[0, \mathrm{~T}] \rightarrow \mathrm{H}_{0}^{1}(\Omega)$, tal que $\frac{\mathrm{d}}{\mathrm{dt}}\left(\mathrm{u}^{\prime}(\mathrm{t}), \mathrm{v}\right)+\mathrm{a}(\mathrm{u}(\mathrm{t}), \mathrm{v})=(\mathrm{f}(\mathrm{t}), \mathrm{v})$ $\forall \mathrm{v} \in \mathrm{H}_{0}^{1}(\Omega)$ en sentido de $\mathrm{D}^{\prime}(0, \mathrm{~T})$. La solución generalizada $\mathrm{u}:[0, \mathrm{~T}] \rightarrow \mathrm{H}_{0}^{1}(\Omega)$ del problema hiperbólico es única.

\section{REFERENCIAS BIBLIOGRÁFICAS}

ADAMS, R.A. (1975).Sobolev Space.Nueva York: Academic Press.

BRÉZIS, H. (1984). Análisis Funcional. Madrid: Alianza Editorial.

MEDEIROS, L. A. (1990). Ecuaciones diferenciales de evolución. Brasil: IMUFRJ.

Ortiz, A. (1988).Aspectos básicos en ecuaciones en derivadas parciales, Departamento de Matemática de la Universidad Nacional de Trujillo: 124-142.Print.

VERA, E. (1984), Una introducción a las ecuaciones diferenciales parciales. Lima: Sociedad Matemática Peruana Segundo Coloquio. 